\title{
Exploring student understanding of the engineering design process using distractor analysis
}

\author{
Stefanie A. Wind ${ }^{1 *}\left(\mathbb{D}\right.$, Meltem Alemdar ${ }^{2}$, Jeremy A. Lingle ${ }^{2}$, Roxanne Moore ${ }^{2}$ and Abdullah Asilkalkan
}

\begin{abstract}
Typical approaches to assessing students' understanding of the engineering design process (EDP) include performance assessments that are time-consuming to score. It is also possible to use multiple-choice (MC) items to assess the EDP, but researchers and practitioners often view the diagnostic value of this assessment format as limited. However, through the use of distractor analysis, it is possible to glean additional insights into student conceptualizations of complex concepts. Using an EDP assessment based on MC items, this study illustrates the value of distractor analysis for exploring students' understanding of the EDP. Specifically, we analyzed 128 seventh grade students' responses to $20 \mathrm{MC}$ items using a distractor analysis technique based on Rasch measurement theory. Our results indicated that students with different levels of achievement have substantively different conceptualizations of the EDP, where there were different probabilities for selecting various answer choices among students with low, medium, and high relative achievement. We also observed statistically significant differences $(p<0.05)$ in student achievement on several items when we analyzed the data separately by teacher. For these items, we observed different patterns of answer choice probabilities in each classroom. Qualitative results from student interviews corroborated many of the results from the distractor analyses. Together, these results indicated that distractor analysis is a useful approach to explore students' conceptualization of the EDP, and that this technique provides insight into differences in student achievement across subgroups. We discuss the results in terms of their implications for research and practice.
\end{abstract}

\section{Introduction}

Researchers have found that engineering education in middle school is essential to increase students' awareness of engineering as a career path; therefore, researchers have identified middle school as a crucial period for engineering education (Tafoya et al. 2005). Further, engineering design is a core component of middle school engineering curricula. As a result, effectively measuring middle school students' understanding of the engineering design process is essential.

Approaches to assessing students' understanding of the engineering design process (EDP) are varied in research and practice. Many of the methods are qualitative in nature. For example, several researchers have used interviews with both students and teachers to

\footnotetext{
* Correspondence: swind@ua.edu

${ }^{1}$ Educational Studies in Psychology, Research Methodology, and Counseling,

The University of Alabama, Box 870231, Tuscaloosa 35487, AL, USA

Full list of author information is available at the end of the article
}

examine a broad-range of perspectives of the EDP, including understanding of design practices, as well as variants of approaches and perspectives of design. Alemdar et al. (2017a, 2017b) applied student interviews to examine student understandings of the design process, perspectives of the collaborative design experience, and recognition of engineering connections to other content areas. Researchers have also incorporated interviews into phenomenological studies for the purpose of identifying concepts such as successful engineering teaching practices (Adams et al. 2003).

In other studies, researchers have used the process of engineering design itself as a data source through methods such as protocol analysis and ethnographies. Protocol analyses explore student approaches to abbreviated design tasks or student responses to design briefs. Field studies and full ethnographies include observations of students as they approach design challenges in the 
classroom environment. Most often, researchers have used these methods to identify patterns in behavior of participants as they design, either as individuals or as groups. Researchers have used think-aloud protocols with individual students, and they have used analyses of dialog for group-based activities. Generally, researchers use these methods to understand the processes of student design and the student conceptions that underlie their design decisions (Atman et al. 2014).

Researchers also frequently use survey methods for the purpose of individual-level assessment. Specifically, researchers conduct studies with surveys to gather information about students' affective responses to engineering, such as design self-efficacy, the relevance and importance of the EDP, and to identify difficulties that students experience as they approach specific stages or design activities (Atman et al. 2014). Surveys are useful in capturing changes in student perception over time, for example to measure self-efficacy and to determine whether student self-efficacy changes before and after a design experience (Purzer 2011).

Another common data source for assessing EDP knowledge is student-produced classroom artifacts. Artifacts may include students' engineering notebooks or journals, reflective narratives, portfolios, or design products such as prototypes. For example, researchers have examined students' engineering journals to document improvements in design practices over time (Sobek II 2002). Likewise, researchers have analyzed students' engineering portfolios to examine professional engineering identify formation (Eliot and Turns 2011). For these artifacts, researchers apply evaluative rubrics to quantify student learning, measuring such aspects as communication, engineering practice, design, and experiments (Mourtes 1999; Williams 2001).

These assessment techniques are valuable in that they can provide researchers and practitioners with detailed insight into students' understanding of engineering design. However, in the classroom, the demands of teaching many students (particularly in secondary schools) often prevent teachers' thoughtful analysis of these products. In contrast, multiple-choice (MC) items can be scored more quickly, and the results can be incorporated more readily into teachers' instructional decisions (Briggs et al. 2006). A common criticism of assessments based on $\mathrm{MC}$ items is that they provide limited diagnostic information regarding students' conceptualization of complex concepts (Klassen 2006). However, it is possible to construct MC items such that analyses of students' answer choices provide insight into students' conceptualizations of the assessed concept (Haladyna and Rodriguez 2013). In particular, distractor analysis is an approach to examining response patterns on MC items in which students' answer choices are examined alongside measures of achievement. When MC items are constructed such that the distractors are related to important aspects of student understanding of a particular concept, this analytic approach provides valuable insight into the types of correct and alternative conceptions that are present within a sample of students, as well as insight into the developmental hierarchy of the misconceptions included within the item distractors. This information can be used to inform instructional decisions to target specific areas of student understanding without the use of more time-intensive performance assessment procedures. Researchers have discussed and applied distractor analysis techniques in many different content areas, including science and engineering (discussed further below). However, there has been relatively limited research on the use of distractor analysis in engineering education for middle school students, and limited use of this approach to evaluate students' understanding of the EDP. Furthermore, few researchers have used distractor analysis to explore differences in student conceptualizations across subgroups.

\section{Purpose}

The primary purpose of this study is to illustrate the diagnostic value of distractor analysis for exploring middle school students' (grades 6-8) understanding of the EDP. As a secondary purpose, we use distractor analysis to explore differences in student achievement across subgroups. Accordingly, we focus on the following research questions:

1. What does distractor analysis of multiple-choice EDP assessment items reveal about students' conceptions of the EDP?

2. How can distractor analysis of multiple-choice EDP assessment items be used to understand differences in student performance on individual items across learning environments?

\section{Distractor analysis as a diagnostic tool}

Despite the limitations that are commonly associated with assessments based on multiple-choice (MC) items, many practitioners and researchers use assessments with $\mathrm{MC}$ items in educational settings. One reason is that $\mathrm{MC}$ items are easy to implement and score objectively, thus allowing test users to assess a wide range of content in a single administration of an assessment. Furthermore, it is possible to construct MC items such that they provide test users with useful diagnostic information about student understanding (Gierl et al. 2017; Thissen et al. 1989). Gierl et al. (2017) conducted a comprehensive literature review of the use of MC item distractors in education, and concluded that, when constructed with diagnostic purposes in mind and analyzed appropriately, $\mathrm{MC}$ item distractors provide useful diagnostic information.

In their review, Gierl et al. (2017) described two major categories of distractor analysis techniques that 
researchers and practitioners can use to extract diagnostic information from $\mathrm{MC}$ items whose answer choices are constructed to provide meaningful diagnostic information, such as alternative conceptions or developmental stages related to a particular concept. Gierl et al. called the first type of distractor analysis traditional distractor analysis. Traditional distractor analysis techniques can be conducted using either classical test theory (CTT) or item response theory (IRT) techniques. In both CTT and IRT traditional distractor analyses, researchers and practitioners examine patterns of students' responses at different total scores (CTT) or achievement estimates (IRT) in order to identify the relationship between students' understanding of the particular concept included in the item, including students' tendency to select particular incorrect answer choices, and students' overall achievement. Such information can be useful for informing theory related to students' development related to a concept or set of concepts as well as for planning or revising instructional activities. Traditional distractor analysis techniques also include analyses of differential distractor functioning (DDF). DDF analyses provide valuable information about the degree to which individual distractors function in the same way for student subgroups given the same achievement level (Terzi and Suh 2015; Penfield 2008). Along with differential item functioning (DIF) analyses, DDF can be used for a variety of purposes, including refining existing tests, developing new instruments, and providing validity evidence for the interpretation and use of test score inferences.

Second, Gierl et al. described contemporary distractor analysis as distractor analysis techniques that are based on relatively more-recent psychometric developments, with the goal of obtaining diagnostic information about students' understanding using their responses to MC items. In particular, some researchers have integrated cognitive diagnostic methods (CDM) with the construction and analysis of $\mathrm{MC}$ item distractors. In traditional multiple-choice educational tests, the purpose of distractors is just to identify students with limited understanding or possible misconceptions of the concept being tested. Among these techniques include the use of two-tier items (Treagust 1995). Two-tier items include traditional MC items that are paired with followup, open-ended items that ask students to explain why they selected their answer. Researchers have used the two-tiered item approach to explore student misconceptions (Treagust 1995) as well as to develop new distractors for MC items (Hestenes et al. 1992). Another example of a contemporary distractor analysis approach is the use of ordered multiple-choice items (Briggs et al. 2006). When test developers construct ordered MC items, they use a learning model to develop distractors that reflect ordinal, increasing levels of understanding of a particular concept. Then, responses to ordered MC items can be analyzed using IRT models in which partial credit is assigned to distractors relative to their proximity to the correct response, such as the ordered partition model (OPM; Wilson 1992). The distinguishing feature of the OPM is that multiple answer choices can share the same score. Similar to the OPM, Wang (1998) proposed a distractor model that assigns different levels of difficulty to each distractor and examines model-data fit statistics associated with the distractors. As a final example of contemporary distractor analysis techniques, Gierl et al. described de la Torre's (2009) modified version of the multiple-choice deterministic-input noisy "and" gate model (DINA; Junker and Sijtsma 2001). Essentially, researchers use this analytic approach to model student responses to $\mathrm{MC}$ items in order to estimate a profile of "attributes" for each student that represent the particular knowledge, skills, or cognitive processes that the student used to arrive at a particular answer choice.

\section{Distractor analysis in engineering education}

As the above review demonstrates, research related to distractor analysis techniques is relatively common in research on educational measurement in general (Gierl et al. 2017). Several researchers have also examined the use of distractor analysis techniques in the context of engineering education. For example, two teams of engineering education researchers proposed frameworks related to the development and evaluation of curriculum and assessment that incorporate distractor analysis as a key component.

First, Jorion et al. (2015) presented an analytic framework for evaluating concept inventories. Concept inventories are popular assessment formats in a variety of domains, including Science, Technology, Engineering, and Mathematics (STEM). These assessments incorporate common or developmentally meaningful misconceptions as a method for identifying and addressing these misconceptions in instructional settings. In their framework, Jorion et al. present distractor analysis as a key component of a body of evidence that researchers and practitioners can collect to provide support for the interpretation and use of assessment instruments. These researchers provided empirical illustrations in which they applied their analytic framework to three assessments in the context of engineering. Within Jorion et al.'s framework, the major role of distractor analysis is to provide insight into student misconceptions to inform curricula and instruction. Importantly, these researchers note that one cannot meaningfully interpret the results from distractor analyses unless there is sufficient evidence that the assessment instrument is targeting the intended domain. These researchers discussed distractor 
analysis techniques based on CTT that reflect the traditional distractor analysis approach, as defined by Gierl et al. (2017). Similarly, Kong et al. (2014) proposed a framework to guide the design of curriculum and assessment. Focusing on nanotechnology as a content area, these researchers presented distractor analysis as a method through which researchers and practitioners can evaluate and improve assessment instruments made up of $\mathrm{MC}$ items, as well as a tool for identifying areas for improvements to engineering curricula. Kong et al. illustrated their framework using empirical data from a MC assessment of nanotechnology concepts. Similar to Jorion et al. (2015), these researchers discussed traditional distractor analysis techniques based on CTT.

In addition to these frameworks, several researchers have used distractor analyses in applied research as a diagnostic method for improving instructional practices. For example, Wage et al. (2005) applied traditional distractor analysis techniques based on CTT to assessments in an undergraduate signals and systems course. These researchers used distractor analysis to identify the most common misconceptions, as well as misconceptions that persisted between a pre-test and post-test. More recently, Peuker et al. (2013) applied traditional distractor analysis techniques based on CTT to an assessment in an undergraduate introductory engineering course. Their study included a comparison of the benefits of constructed response (CR) and MC-format assessments for informing instructional practices in engineering education. These researchers concluded that distractor analysis "can give insight to student misconceptions in the same way that the traditional CR problems can provide the instructor information about misunderstandings," and provide an efficient means through which to incorporate this information into instruction (p. 9). Along the same lines, Sengupta et al. (2017) used traditional distractor analysis techniques based on CTT to examine a concept inventory in an undergraduate environmental engineering course. These researchers discussed the benefits of distractor analysis as a tool for improving the quality of $\mathrm{MC}$ items, as well as a method for improving instructional practices.

In this study, we use a traditional, Rasch-based application of distractor analysis to examine students' conceptual understanding of the EDP. We also extend previous research on distractor analysis in general by illustrating a method for using Rasch-based distractor analysis to explore differences in patterns of student responses to $\mathrm{MC}$ items related to student subgroups.

\section{Methods}

In order to explore the research questions for this study, we used data from middle-school students' responses to a MC EDP assessment that was administered as part of an experimental engineering curriculum project. After we examined the quantitative data, we also examined qualitative data from a later study of the same student participants in order to more fully understand the quantitative results. The quantitative data were our primary focus in the analysis, and the qualitative data served a secondary, explanatory role. We collected and analyzed the quantitative and qualitative data separately, and then merged the qualitative results with our quantitative results. Thus, our study can be described as a convergent explanatory design where the quantitative results were primary (Creswell and Plano Clark 2011). We describe the context for data collection, the instruments, and our data analysis methods below.

\section{Context for data collection}

Our data were collected during the implementation of an 18-week STEM-Innovation \& Design (STEM-ID) engineering curriculum for middle school students. ${ }^{1}$ Curriculum developers iteratively designed the courses based on feedback from teachers and observations of the course in action (Alemdar et al. 2017a, 2017b). The courses were designed around the framework of the engineering design process (EDP). Essentially, the EDP is a conceptual model for the application of engineering principles to practical design problems. Researchers and practitioners have used a variety of EDP models as guiding frameworks for engineering education that vary in terms of specific terms and sequences of activities (Carberry et al. 2010). Figure 1 includes the specific EDP that was used in the curriculum context from which our data were collected; we provide operational definitions for each stage in our EDP model in Additional file 1. The engineering curriculum was designed to utilize the EDP within a problem-based learning context, combined with an emphasis on science and mathematics practices, as defined by the Next Generation Science Standards (NGSS; NGSS Lead States 2013) and the Standards of Mathematical Practice (Illustrative Mathematics 2014).

\section{Instruments}

\section{Multiple choice engineering design process assessment}

A team of engineering education experts and psychometricians developed a series of MC EDP assessments for each grade level (6th, 7th, and 8th) that corresponded to the experimental curriculum. The assessments were developed iteratively using a mixedmethods approach (Alemdar et al. 2017a; Wind et al. 2017). Each of the EDP assessment items was aligned with one or more stages of the EDP (see Fig. 1 and Additional file 1). Furthermore, the items were developed such that the distractors reflected alternative conceptions related to engineering in general or particular stages in the EDP that could be addressed with 


\section{Engineering Design Process}

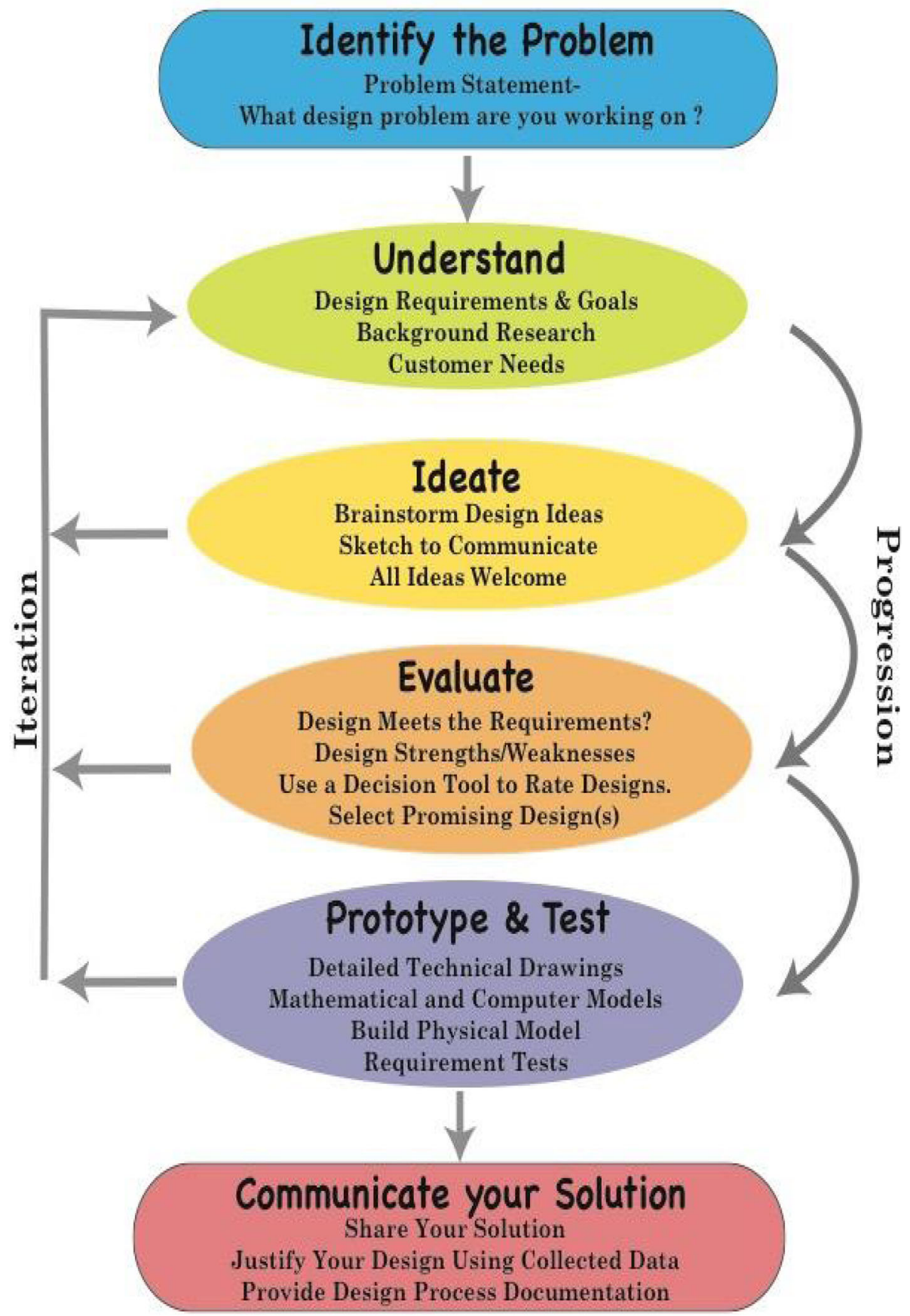

Fig. 1 Conceptual model for the engineering design process. This figure is the representation of the engineering design process included in the curriculum used to instruct the students who participated in this study

additional instruction (for details about the development and students' interpretations of the distractors, please see Alemdar et al. 2017a, 2017b and Wind et al. 2017). Results from previous analyses suggested that the final versions of the EDP assessments demonstrated acceptable psychometric properties, such that they could be used to evaluate student achievement following the implementation of the experimental engineering curriculum (see Wind et al. 
2017). We provide additional background information about the EDP assessment used in this study, as well as the context in which we collected data in Additional file 1.

\section{Student interview protocol}

After we collected student responses to the MC items, we collected supplementary qualitative data to inform our interpretation of the quantitative results. Specifically, we conducted semi-structured interviews with students using an interview protocol. All of the interviewers used the same semi-structured interview protocol with follow-up questions to elicit rich data that could be subjected to sequential qualitative analysis (Miles et al. 2014). We designed the interview protocol around several themes, including students' understanding of the engineering design process, their perceptions about the course, and the mathematics and science connections. In this study, we focused on interview results related to students' understanding of the EDP. Specifically, during the relevant section of the interview, we asked students to describe the use of the EDP while working on a design challenge. Some of the questions included the following: "Tell me about how you used the Engineering Design Process in your STEM-ID class. Were you ever confused about what you were supposed to do for a certain stage in the engineering design process?"

\section{Participants}

In the current analysis, we focused on the Fall 2016 administration of the EDP assessment for seventh grade students, which included 20 items. A total of 128 students participated in this assessment who were enrolled in four classrooms in separate schools (teacher 1: $N=39$; teacher 2: $N=25$; teacher 3: $N=35$; teacher 4: $N=29$ ). These sample sizes are within the recommended range for Rasch analyses of low-stakes multiple-choice assessments for stable estimates of item or person calibrations within \pm 1 logit over replication (Linacre 1994; Wright and Stone 1979). Using the interview protocol described above, we collected qualitative data from thirty students from four middle schools.

\section{Data analysis}

In order to explore the use of distractor analysis as a method for understanding students' conceptualizations of the EDP, we used a combination of quantitative and qualitative analytic tools. In light of the purpose of our study, we focused primarily on the results from the quantitative data analysis, and we used qualitative data to inform our interpretations of the quantitative results.

\section{Quantitative data analysis}

Our quantitative data analysis procedure consisted of four main steps. First, we calculated measures of student achievement and item difficulty using the dichotomous Rasch model (Rasch 1960). Briefly, the Rasch model is a statistical approach that can be used to examine student responses to items in educational assessments in order to obtain measures of student achievement and item difficulty. This approach is based on a transformation of assessment item responses from an ordinal scale to a log-odds (logit) scale, such that measures of student achievement and item difficulty can be expressed on a common linear scale with equal units.

We selected the Rasch model for our analysis for three reasons. First, this model has been used in previous applications of distractor analysis techniques in STEM education (Herrmann-Abell and DeBoer 2011, 2014; Wind and Gale 2015). Second, the EDP assessment was developed based on the Rasch model (Wind et al. 2017). Finally, the Rasch model leads to a straightforward interpretation of student achievement and item difficulty that facilitates the interpretation of results from the distractor analysis procedure. The model can be stated in log-odds form as follows:

$$
\ln \left[P_{n i}(x=1) / P_{n i}(x=0)\right]=\theta_{n}-\delta_{i}
$$

where $\ln \left[P_{n i(x=1)} / P_{n i(x=0)}\right]$ is the log of the odds that a student $n$ provides a correct response to item $i$, rather than an incorrect response; $\theta_{n}$ is the location of student $n$ on the logit scale; and $\delta_{i}$ is the location of item $i$ on the logit scale. Student measures reflect student achievement levels, where higher values indicate higher achievement, and lower values indicate lower achievement. Likewise, item locations reflect item difficulty. For items, higher locations suggest more difficult items, and lower locations suggest easier items. We conducted the Rasch analysis using the Facets ${ }^{\mathrm{Tm}}$ software program (Linacre 2015).

In step two, we examined students' responses to the EDP assessment items using distractor analysis. We deigned our distractor analysis procedure based on a study by Wind and Gale (2015). Specifically, we started by examining the frequency of students who selected each of the four answer choices for all 20 EDP assessment items in order to verify that at least one student selected each of the answer choices for all items. Evidence that students used all four of the answer choices suggested that the distractors are useful for exploring patterns in student understanding. We also examined the average achievement level ( $\theta$ estimates from Eq. 1) among students who selected each answer choice. If the item was functioning as expected, the correct answer choice would be associated with the highest average achievement level. 


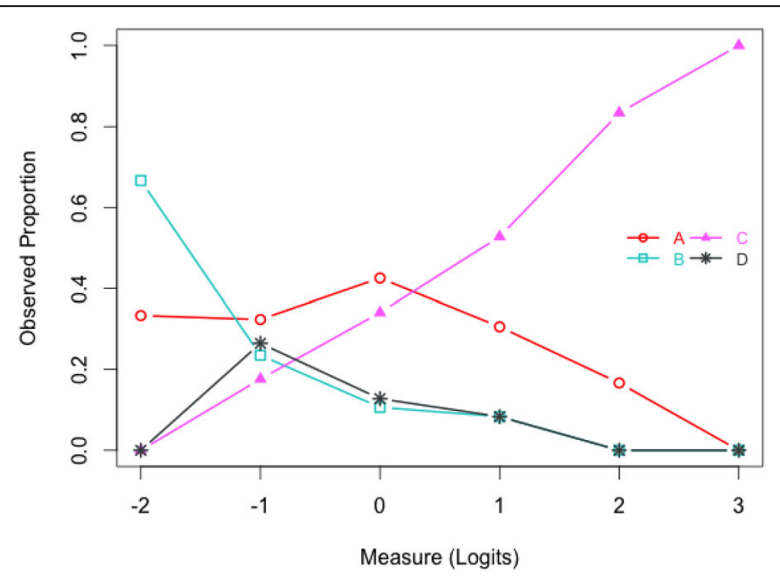

Fig. 2 Example distractor analysis plot. This figure shows an example of a distractor analysis plot for a multiple-choice item. The $x$-axis displays estimates of student achievement. Student achievement estimates are shown in logits, and these estimates were calculated using the Rasch model. Low values indicate lower achievement, and high values indicate higher achievement. The $y$-axis shows the observed proportion of responses for a given answer choice. The lines in the plot show the proportion of students who selected each of the four answer choices (A, B, C, or D) at each level of achievement. For this example item, the correct response is $C$ (pink line with triangle plotting symbols)

To interpret the distractor analysis results, we constructed graphical displays that illustrate the relationship between measures of student achievement and the probability of selecting each of the four answer choices. Figure 2 is an example of a distractor analysis plot calculated using student achievement estimates from the Rasch model. The $x$-axis shows Rasch-model estimates of student achievement. The lines in the plot show the proportion of students ( $y$-axis) who selected each answer choice, where different lines are used for each answer choice. The correct answer for the example item is C. As might be expected, the proportion of students selecting answer choice $C$ increased across increasing levels of student achievement. One can obtain additional information about students' answer choices by examining the proportion of students who selected the three incorrect answer choices in relation to student achievement. Specifically, beginning with the lowestachieving students (farthest left on the $x$-axis), students with the lowest overall achievement levels were mostoften attracted to answer choice B, followed by answer choice A. Moving to the right, students with slightly higher achievement levels were most often attracted to answer choice A. Answer choice A remained the most popular distractor for all other achievement levels.

In step three, we identified items on the EDP assessment on which students in the four classrooms had significantly different performance using an extension of the Rasch model that included an additional variable for classrooms. Specifically, we specified a Many-Facet Rasch (MFR) model (Linacre 1989) with a facet for classrooms as follows:

$$
\ln \left[P_{n i g(x=1)} / P_{n i g(x=0)}\right]=\theta_{n}-\delta_{i}-\eta_{g}
$$

where $\theta_{n}$ and $\delta_{i}$ are defined as in Eq. 1 , and $\eta_{g}$ is defined as the average achievement level of students within classroom $g$. We estimated this model using Facets (Linacre 2015).

Using Eq. 2, we examined differences in student achievement across classrooms related to individual items. Specifically, we used the Facets computer program to calculate the difficulty of each EDP assessment item separately for each classroom. Then, we used pairwise analyses ( $t$ tests) to determine whether the difficulty of individual items were significantly different across the classrooms. This test is appropriate for comparing item estimates between classrooms because the item estimates are calculated on an interval-level scale.

Finally, in step four, we constructed distractor plots separately for the classrooms between which we observed significant differences in item difficulty. After constructing the graphical distractor analysis plots, we used substantive theory related to the EDP, information from the curricular context, and results from the qualitative analyses (described below) to interpret the patterns revealed by the distractor plots.

\section{Qualitative data analysis}

We used the NVIVO $^{\text {rm }}$ software program to code and analyze the student interviews according to a sequential qualitative analysis process (Miles et al. 2014). We completed qualitative coding in two phases. In the first phase, a single coder categorized student responses according to the major topic areas included in the interview protocol. In the next phase, a group of four coders applied a provisional list of sub-codes to a cross-sectional sample of interview transcripts. These sub-codes included process codes aligned to individual phases of the EDP (e.g., problem identification, prototyping, and testing solutions) and magnitude codes indicating positive and negative examples.

\section{Mixed-methods analysis}

After we collected and analyzed the quantitative and qualitative data, we merged the results from the qualitative analysis with the results from our quantitative analyses. Specifically, we used the qualitative interview data to inform our interpretation of step two (distractor analysis for EDP items based on the complete sample) and step four (distractor analysis across teachers) of our quantitative analyses. 


\section{Results}

Step one: overall calibration of the EDP assessment

Before we conducted our analyses, we checked the Rasch model assumptions of unidimensionality (i.e., student responses to the assessment items are primarily due to only one latent variable), and local independence (i.e., after controlling for the latent variable of interest, there are no systematic relationships or dependencies among students' responses to the items). We checked the unidimensionality assumption using a principal components analysis of the residuals, or differences between the observed responses and the responses that would be expected based on the Rasch estimates (Linacre 1998). This analysis allowed us to check for any meaningful patterns in residuals that suggest that more than one latent variable significantly contributed to student responses. We checked the local independence assumption by calculating correlations between the residuals associated with each item.

In the combined sample (all of the classrooms), the Rasch measures explained $22.23 \%$ of the variance in responses; this value is evidence of acceptable adherence to the assumption of unidimensionality for Rasch analyses, because it is higher than the generally accepted critical value of $20 \%$ for analyzing potentially multidimensional instruments (Reckase 1979). Furthermore, we examined the inter-item correlations of model residuals within the combined sample; these values were less than 0.30. We also observed adherence to the Rasch assumptions within the individual school sites. Specifically, the Rasch measures explained $>20 \%$ of the variance in

Table 1 Summary statistics from the dichotomous Rasch model

\begin{tabular}{lll}
\hline Statistic & Student $(N=128)$ & Item $(N=20)$ \\
\hline Location (logits) & 0.11 & 0.00 \\
$M$ & 0.92 & 0.63 \\
SD & & \\
Standard error & 0.52 & 0.20 \\
$M$ & 0.07 & 0.01 \\
SD & & \\
Infit MSE & 1.00 & 1.00 \\
$M$ & 0.14 & 0.14 \\
SD & & \\
Outfit MSE & 1.01 & 1.01 \\
$M$ & 0.22 & 0.21 \\
SD & & \\
Separation statistics & 0.68 & 0.90 \\
Reliability of separation & $332.7^{*}$ & $181.7^{*}$ \\
Chi-Square & 127 & 19 \\
Degrees of freedom & & \\
\hline${ }^{*} p<0.05$ &
\end{tabular}

${ }^{*} p<0.05$ students' responses for each of the classrooms. Furthermore, the inter-item correlations of model residuals were less than 0.30 for all of the classrooms. Because all of our sub-samples approximated the Rasch model assumptions, we proceeded with our analyses.

Table 1 summarizes the results from the first step of the data analysis procedure. The average estimated student measure was $\theta=0.11(S D=0.92)$, which is near the average estimated item difficulty location (mean $\delta=0.00 ; S D=0.63$ ). This result suggests that the assessment was targeted well to the group of seventh grade students. The relatively low standard errors (SE) for student estimates $(M=0.52, S D=0.07)$ and item estimates $(M=0.20, S D=0.01)$ provide additional evidence of precision for the Rasch estimates. The average student $\mathrm{SE}$ is larger than the average item SE because there are more observations of each item (128 students responded to each item) compared to the observations of each student (each student is observed using 20 items). Nonetheless, these SEs are within the ranges that other researchers have reported for low-stakes MC tests in a variety of educational domains (Bond and Fox 2015).

Furthermore, average values of model-data fit statistics are within the range of values that previous researchers have established as expected when there is acceptable fit to the Rasch model (MSE fit statistics around 1.00; Smith 2004; Wu and Adams 2013). For items, the Rasch reliability of separation statistic indicated significant differences in difficulty across the individual items $(\mathrm{Rel}=0.90)$; however, the reliability of separation statistic for students was notably lower ( $R e l=0.60)$, suggesting that there were several groups of students who had similar levels of achievement. Taken together, the practical implication of these results is that the distractor analyses can be meaningfully interpreted in terms of students' understanding of the EDP.

\section{Step two: distractor analysis for EDP items based on the complete sample}

For all 20 items, the correct response was associated with the highest average achievement level. These results suggest that the answer choices for the MC EDP items were functioning as intended. Accordingly, we examined students' responses to the EDP items using distractor analysis plots. We illustrate the results from this step using four example items: item 12, item 14, item 17, and item 19. We selected these example items for two main reasons. First, they represent different stages of the EDP. Second, these items provide clear examples of the types of patterns of student answer choices that we observed over all 20 items. 


\section{Item 12}

Item 12 was developed to assess students' understanding of the Identify the Problem stage of the EDP, as well as their general understanding of the EDP as a process. To answer this item, students had to consider a scenario in which they were asked to design a new oven for a company that makes ovens for restaurants and bakeries, and identify the first step in this process according to the EDP. We present the text and distractor analysis plot for item 12 in Fig. 3. Examination of the plot reveals several interesting aspects of student understanding related to the first stage of the EDP. First of all, the proportion of students who selected the correct response $(D)$ increased across increasing levels of student achievement-suggesting that the highest-achieving students had the expected understanding of this stage of the EDP. Next, it is interesting to note that the second-most prominent answer choice was distractor B, followed by distractor $\mathrm{C}$. This pattern suggests that students who did not select the correct answer appeared to skip ahead in the EDP to the Ideate stage, as reflected in answer choice B, or the Prototype $\mathcal{E}$ Test stage, as reflected in answer choice C.

\section{Item 14}

Item 14 was developed to assess students' understanding of the Evaluate and the Communicate your Solution stages of the EDP. This item asked students to identify relevant design details to communicate to the manager of an oven company (see Fig. 4). As expected, the proportion of students who selected the correct response $(D)$ increased across increasing achievement levels; likewise, students were less attracted to each of the distractors as achievement levels increased. Among those students who selected incorrect responses, students selected distractor A most often, followed by distractor $B$. Inspection of the three most popular answer choices (D (correct response), A, and B) indicates that students recognized that a company manager may not be interested in results from early stages of the EDP, as reflected in distractor $C$, but had some difficulty determining which of the other details would be most relevant for the specified customer. The results from our qualitative analyses of student interviews reflected similar findings. For example, one student described the Communicate your Solution stage as follows: "Communicating your solution, like thinking of what to say, specific things you have to think and to tell other people to get your product. That's really hard, too." In general, student responses to the interview questions suggested that students have a general grasp of what is needed to communicate with a client, but that this step is difficult.

\section{Item 17}

Item 17 was intended to evaluate students' understanding of the Understand stage of the EDP. This item asked students to determine an appropriate next step after learning that customers do not prefer the visual characteristics of an oven design (see Fig. 5). As expected, the proportion of students who selected the correct response (B) increased across increasing achievement levels, suggesting that high achieving students had the expected understanding of this stage of the EDP. Across the range of achievement levels, students who provided incorrect responses to this item selected distractor $C$ most

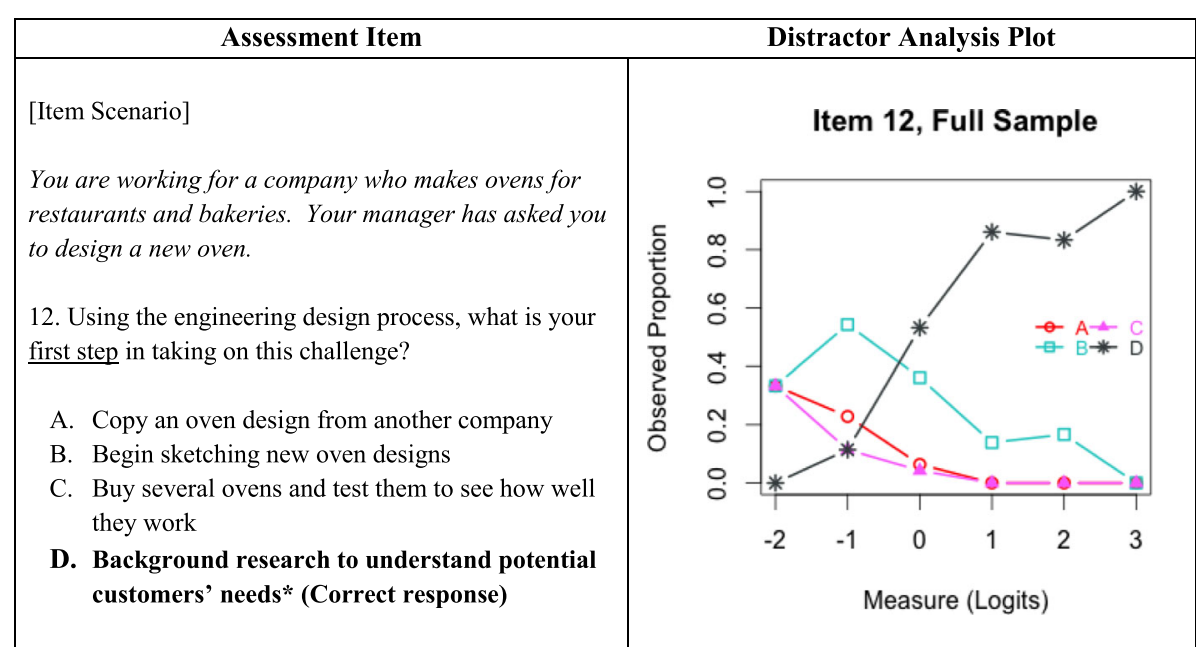

Fig. 3 Distractor analysis plot for item 12: full sample. The plot shows patterns of student answer choices for item 12 within the entire sample. The $x$-axis displays estimates of student achievement. Student achievement estimates are shown in logits, and these estimates were calculated using the Rasch model. Low values indicate lower achievement, and high values indicate higher achievement. The $y$-axis shows the observed proportion of responses for a given answer choice. The lines in the plot show the proportion of students who selected each of the four answer choices (A, B, C, or D) at each level of achievement. For this item, the correct response is D (black line with asterisk plotting symbols) 


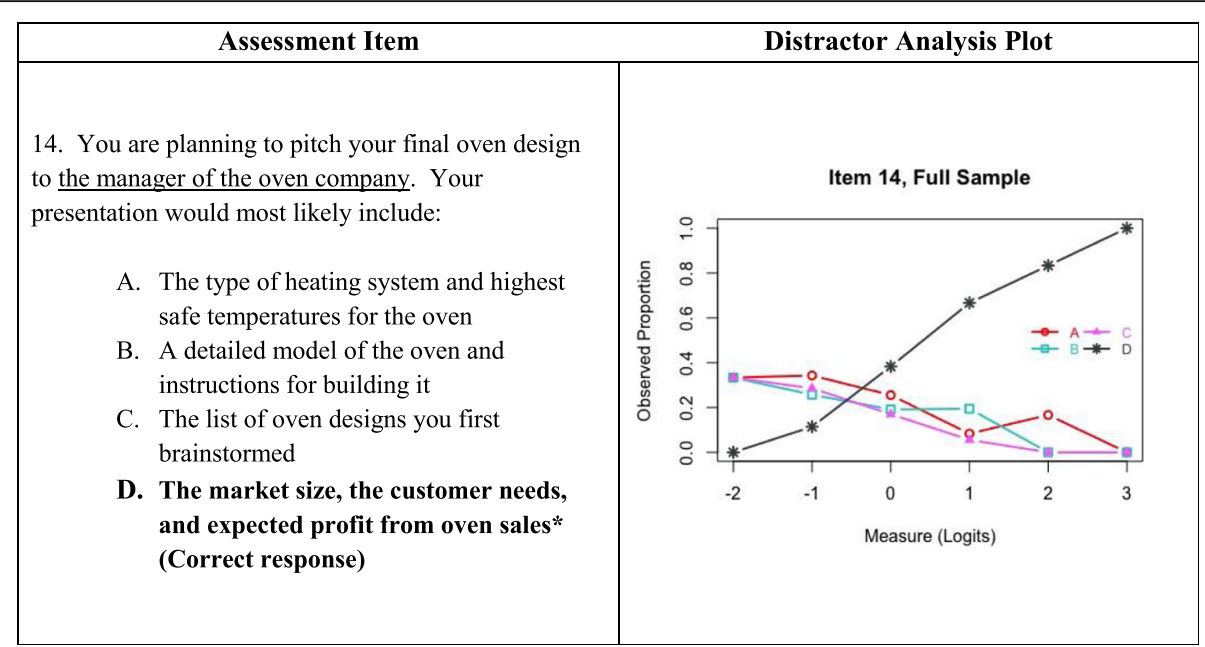

Fig. 4 Distractor analysis plot for item 14: full sample. The plot shows patterns of student answer choices for item 14 within the entire sample. The $x$-axis displays estimates of student achievement. Student achievement estimates are shown in logits, and these estimates were calculated using the Rasch model. Low values indicate lower achievement, and high values indicate higher achievement. The $y$-axis shows the observed proportion of responses for a given answer choice. The lines in the plot show the proportion of students who selected each of the four answer choices (A, B, C, or D) at each level of achievement. For this item, the correct response is D (black line with asterisk plotting symbols)

often. Examination of the other distractors indicates that most of the students recognized that the appropriate next step according to the EDP involved revising the design of the oven, as reflected in distractor C. Students who selected distractor $C$ focused on the performance of the oven rather than the visual characteristics of the design.

\section{Item 19}

Item 19 was intended to assess students' understanding of the Evaluate stage of the EDP. This item asked students to identify an activity that reflects the Evaluate stage of the EDP with regard to the design of a recycling bin for a school cafeteria (see Fig. 6). The correct answer for this item is C. Compared to the previous example items, students' responses to item 19 were more evenly distributed among all four answer choices. Although the highestachieving students selected the correct response most often, the lowest-achieving students $(\theta<0)$ were equally attracted to the three distractors. Students with middlerange achievement $(0 \leq \theta \leq 1)$ selected distractor $\mathrm{B}$ most

\begin{tabular}{|l|l|}
\hline \multicolumn{1}{|c|}{ Assessment Item } \\
17. Through market research you find that restaurants \\
do not like the way the ovens look. After updating \\
your requirements, what should you do for your next \\
version of the design?
\end{tabular}

Fig. 5 Distractor analysis plot for item 17: full sample. The plot shows patterns of student answer choices for item 5 within the entire sample. The $x$-axis displays estimates of student achievement. Student achievement estimates are shown in logits, and these estimates were calculated using the Rasch model. Low values indicate lower achievement, and high values indicate higher achievement. The $y$-axis shows the observed proportion of responses for a given answer choice. The lines in the plot show the proportion of students who selected each of the four answer choices (A, B, C, or D) at each level of achievement. For this item, the correct response is B (turquoise line with square plotting symbols) 


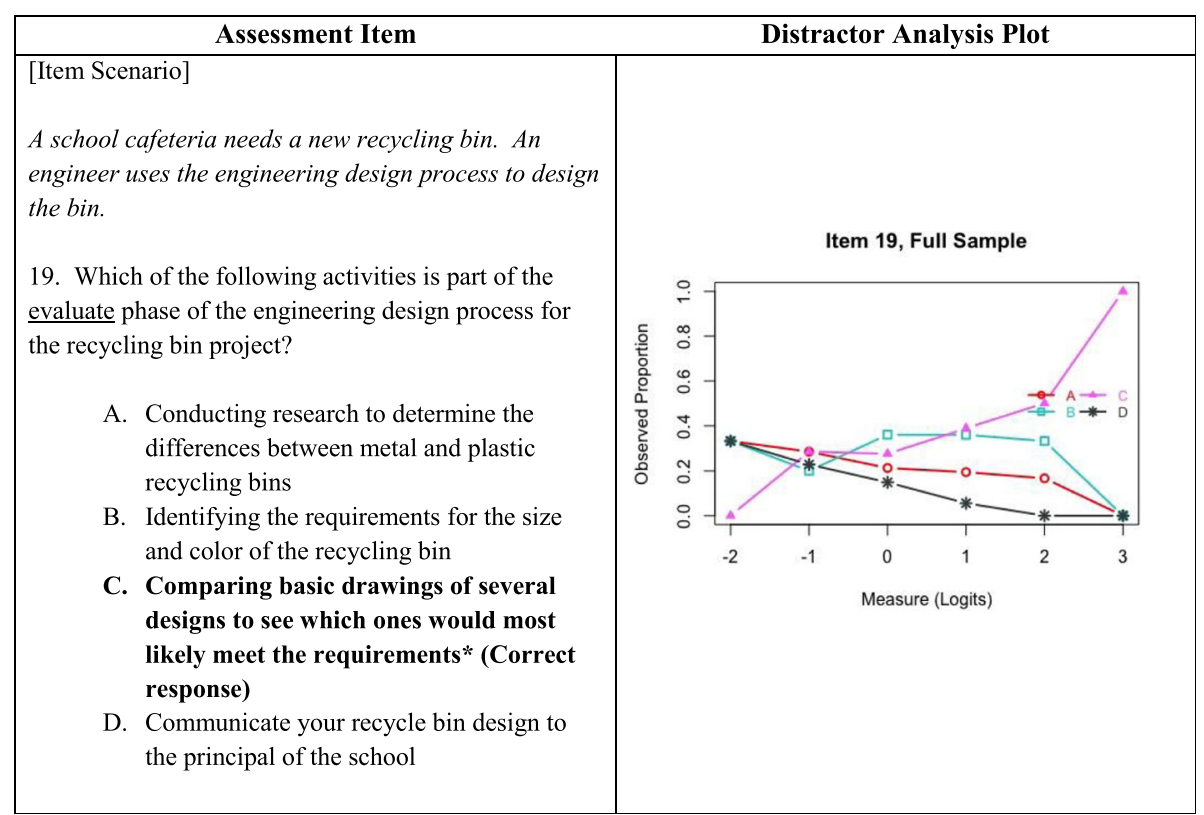

Fig. 6 Distractor analysis plot for item 19: full sample. The plot shows patterns of student answer choices for item 16 within the entire sample. The $x$-axis displays estimates of student achievement. Student achievement estimates are shown in logits, and these estimates were calculated using the Rasch model. Low values indicate lower achievement, and high values indicate higher achievement. The $y$-axis shows the observed proportion of responses for a given answer choice. The lines in the plot show the proportion of students who selected each of the four answer choices (A, B, C, or D) at each level of achievement. For this item, the correct response is C (pink line with triangle plotting symbols)

often. Interestingly, the activity described in distractor B involves aspects of the correct response, in that the engineer considers the requirements for the recycling bin-indicating that students who selected this answer choice may have had some emerging understanding of the Evaluate stage of the EDP. Student responses during the interviews often indicated an understanding of the purpose of the Evaluate stage, which is to choose a design based on how likely it is to meet the requirements. For example, when asked how a wing design was selected from among the potential airplane designs, one student responded: "We decided based on how we would meet your requirements and what would be the best possible choice for it." However, some students did not understand this purpose. For example, in the following quotation, a student explains that the wing design choice was based upon personal preference.

Interviewer: "How did you decide which one you were going to try?"

Student: "Because I've always had a favorite plane. You know the planes that they got the low wings and those little standup things on the end? That's my favorite plane. I don't know what it's called but it's my favorite plane. I always do that one. That's how I chose my plane."

Together, our qualitative analyses of student interview responses corroborate our quantitative results. That is, the students who did not fully understand the purpose of the Evaluate stage of the EDP demonstrated some confusion with regard to the purpose of this stage, which may have led them to select incorrect answer choices.

\section{Step three: items with different difficulty levels across classrooms}

The third step in our data analysis procedure was to identify differences in item difficulty across the four classrooms of students who participated in the administration of the EDP assessment using the MFR model (Eq. 2; Linacre 1989). We observed seven items for which there were statistically significant differences in difficulty between at least two classrooms $(p<0.05)$ : items $1,2,3,7,9,16$, and 19.

\section{Step four: distractor analysis across teachers}

For each the items that we identified in step three, we constructed distractor analysis plots separately using the data from students in each of the four teachers' classrooms. We created these teacher-level plots for items for which we observed statistically significant differences in item difficulty. We illustrate our procedure for exploring students' responses separately by teacher using three example items: item 7, item 9, and item 19.

\section{Item 7}

Item 7 was intended to assess students' understanding of the Prototyping $\mathcal{E}$ Testing stage of the EDP. This 
item asked students to identify the stage of the EDP during which engineers create detailed technical drawings for a new design. For this item, we observed statistically significant differences in achievement between students who were instructed by teacher 1 and teacher 2. Accordingly, we constructed distractor analysis plots separately for these two subgroups of students (see Fig. 7). For all of teacher 1's students, the correct response $(\mathrm{C})$ was the most popular answer choice, and no students selected answer choice D. Because distractors A and B reflect earlier stages in the EDP than the correct answer choice (Prototype \& Testing), these results suggest that the students in teacher 1's classroom understood that creating technical drawings is an activity that occurs before the final stages of the EDP. Students' answer choices were more varied in teacher 2's classroom. In this classroom, students with the lowest achievement level were most often attracted to answer choices B and A. Similar to teacher 1's students, none of teacher 2's selected answer choice D. However, it is interesting to note that, for teacher 2's students, the correct response was only the most popular answer choice among students with achievement levels around $\theta=1$. Students with the highest achievement levels $(\theta \geq 1)$ selected answer choice A most often. Results from our qualitative analyses suggested that students' confusion between the Prototyping $\mathcal{E}$ Testing stage and other stages is often due to the students' desire to quickly move through the stages in order to create a prototype, as illustrated by the following quotations from a student interview:

Interviewer: "Have there ever been times when you've skipped any of these stages or noticed other students skipping any of the stages?"

Student: "Someone skipped Ideate in my last class. Theirs just completely failed. Their catapult completely failed. Last year we had to make a game for a carnival, it had to do with a catapult and shooting it. It hit none of the points."
7. Which stage of the engineering design process includes creating detailed technical drawings for a new design?
A. Ideate
B. Evaluate
C. Prototype and test* (Correct response)
D. Share your solution

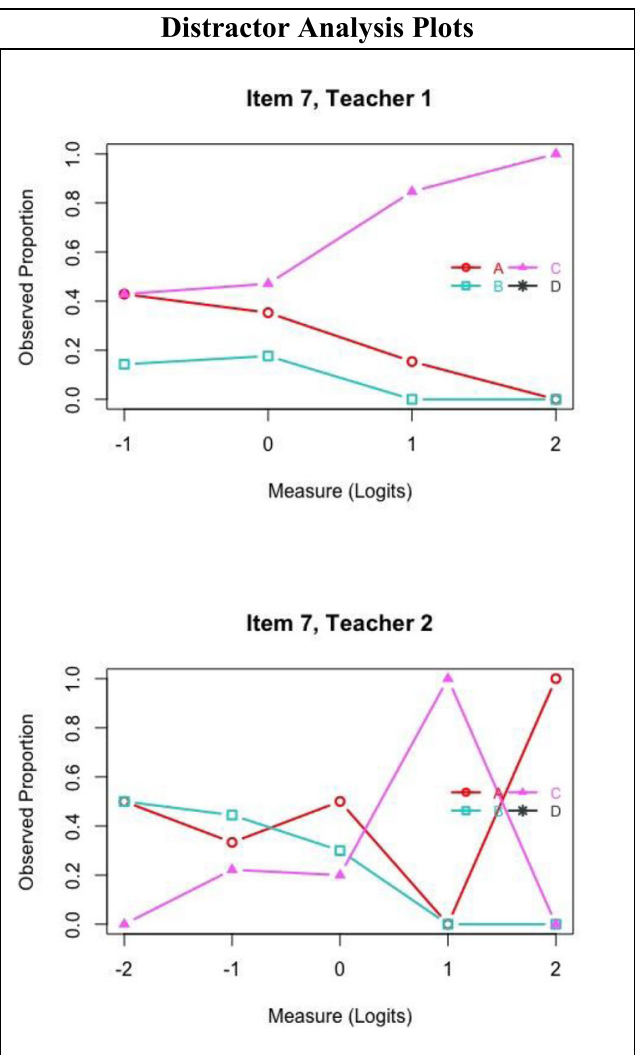

Fig. 7 Distractor analysis plot for item 7: teacher 1 and teacher 2. The plots in this figure show patterns of student answer choices for item 7 in teacher 1's classroom (top plot) and in teacher 2's classroom (bottom plot). In both plots, the $x$-axis displays estimates of student achievement. Student achievement estimates are shown in logits, and these estimates were calculated using the Rasch model. Low values indicate lower achievement, and high values indicate higher achievement. The $y$-axes shows the observed proportion of responses for a given answer choice. The lines in each plot show the proportion of students who selected each of the four answer choices (A, B, C, or D) at each level of achievement. For this item, the correct response is C (pink line with triangle plotting symbols) 
Interviewer: "That's interesting. In your class, how do you know that they skipped that stage?"

Student: "Because after they thought of the idea, they just skipped right into making the prototype."

In this interview excerpt, the student described a situation where their classmates skipped a stage of the EDP-thus corroborating the results from our distractor analysis, which indicated confusion about the sequence of activities in the EDP.

\section{Item 9}

Item 9 was intended to assess students' understanding of the Understand stage of the EDP. This item is based on a scenario where students are asked to consider a set of design requirements for a dog container to be used in airplanes. To answer the item, students were asked to consider a new customer request and determine what the new request represents with regard to the EDP. For this item, we observed statistically significant differences in achievement between students who were instructed by teacher 1 and teacher 2, as well as between the students who were instructed by teacher 1 and teacher 4 . Accordingly, we constructed distractor analysis plots separately for the students who were instructed by teacher 1, teacher 2, and teacher 4 (see Fig. 8). Among teacher 1's students, it is interesting to note that the correct response was the most popular answer choice for the lowest-achieving students, as well as among high-achieving students. However, for students with mid-range achievement ( $\theta$ around 0$)$, distractor $\mathrm{C}$ was most popular. Furthermore, an equal proportion of the highest-achieving students in this classroom selected the correct response (B) as well as distractor A. On the other hand, students in classroom 2 were only attracted to one distractor $(C)$. Finally, among teacher 4's students, the correct response was the most popular for students with all achievement levels. However,

Assessment Item
ABC Airlines has a problem. They have many
customers who want to take their large dogs
with them on vacation, but the airline doesn't
have containers that can safely transport dogs
on long flights. You have been hired as an
engineer to invent a new kind of container to
safely transport dogs in the baggage area in the
belly of the plane. Your container must:
- Allow dogs to have enough air to fly
safely for 8 hours
Be soundproof enough that passengers
cannot hear barking dogs
Be less than 1 meter tall $x$ l meter wide
- 1 meter deep
Cost no more than $\$ 25$
Be non-toxic to dogs
9. After you make your first design, the airline
requests a food dispenser so dogs will be able to
eat on long flights. This is an example of:
A. A tradeoff between the size and the
cost of the container
B. An addition to the requirements
your design needs to address. *
(Correct response)

Fig. 8 Distractor analysis plot for item 9: teacher 1, teacher 2, and teacher 4. The plots in this figure show patterns of student answer choices for item 9 in teacher 1's classroom (top plot), teacher 2's classroom (middle plot), and teacher 4's classroom (bottom plot). In each plot, the $x$-axis displays estimates of student achievement. Student achievement estimates are shown in logits, and these estimates were calculated using the Rasch model. Low values indicate lower achievement, and high values indicate higher achievement. The $y$-axes shows the observed proportion of responses for a given answer choice. The lines in each plot show the proportion of students who selected each of the four answer choices (A, B, C, or D) at each level of achievement. For this item, the correct response is $B$ (turquoise line with square plotting symbols) 
for the lowest-achieving students who did not answer item 9 correctly, distractor A was most attractive. Based on the quantitative results, this item was effective at identifying students who had a sophisticated understanding of the role of requirements in engineering design, specifically with regard to adding a requirement (Understand). In the qualitative data, we identified several students who appeared to combine this stage with activities typically included in the Ideate stage:

Interviewer: What sort of things did you do to understand the problem?

Student: We looked up some websites that were going to help us in the cell phone holder, and we designed some sketches on paper to what we liked.

In this example, the student demonstrated some confusion with regard to the unique characteristics of the Understand stage of the EDP.
Item 19

Our final example of teacher-level distractor analysis is item 19. As we noted above, item 19 was intended to assess students' understanding of the Evaluate stage of the EDP, and the item asks students to identify an activity that is part of this stage. For this item, we observed statistically significant differences in achievement between students who were instructed by teacher 2 and teacher 3 ; accordingly, Fig. 9 includes distractor analysis plots for these subgroups of students. Among teacher 2's students, students with the lowest achievement levels $(\theta$ $<-1)$ were most often attracted to distractors B and A. According to the EDP used in the experimental curriculum, the activities included in these stages precede Evaluate. Accordingly, these students' responses indicate that they were able to eliminate activities associated with the final stages of the EDP, as reflected in answer choice D. Among teacher 3's students who did not provide a correct response to item 19, students with the lowest achievement levels $(\theta \leq-1)$ were attracted to answer

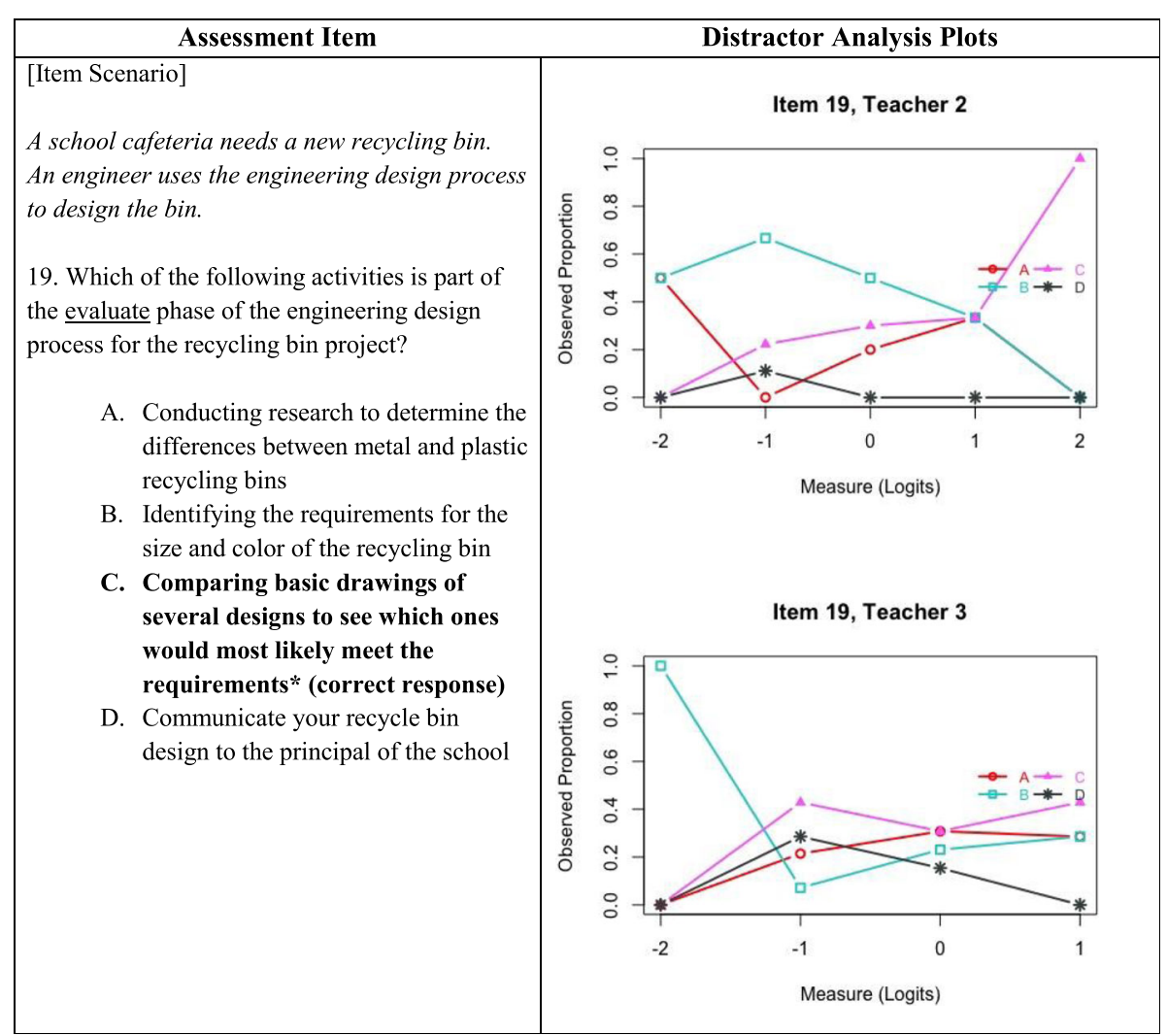

Fig. 9 Distractor analysis plot for item 19: teacher 2 and teacher 3. The plots in this figure show patterns of student answer choices for item 19 in teacher 2's classroom (top plot) and in teacher 3's classroom (bottom plot). In both plots, the $x$-axis displays estimates of student achievement. Student achievement estimates are shown in logits, and these estimates were calculated using the Rasch model. Low values indicate lower achievement, and high values indicate higher achievement. The $y$-axes shows the observed proportion of responses for a given answer choice. The lines in each plot show the proportion of students who selected each of the four answer choices (A, B, C, or D) at each level of achievement. For this item, the correct response is $C$ (pink line with triangle plotting symbols) 
choice B most often. Although the proportion of students selecting the correct response decreased slightly in some intervals across increasing achievement levels, it was the most popular answer choice for students with mid-range and high achievement levels.

\section{Conclusions}

In this study, we used distractor analysis to explore students' understanding of the EDP in the context of an experimental engineering curriculum for middle school students. Specifically, we examined the alignment between students' overall proficiency levels on a multiple-choice EDP assessment and the probability that they selected certain answer choices. Because the assessment items were developed such that the answer choices reflected alternative conceptions related to engineering in general or particular stages in the EDP that could be addressed with additional instruction, patterns of students' responses over different levels of achievement provided insight into the relationship between students' overall achievement and their conceptualizations of the EDP. We also considered differences in patterns of students' responses between subgroups of students. In particular, for items on which we observed differences in student performance, we used distractor analysis techniques to explore further the differences in students' conceptualizations of the EDP that may have contributed to different overall performance between subgroups.

Overall, our results suggested that distractor analysis provides insight into students' conceptualizations of the EDP across different levels of achievement. Our observation that students with different achievement levels selected different incorrect answer choices suggests that students with different levels of overall achievement may have different conceptualizations of the EDP stages. By examining patterns of student responses to individual items, we were able to identify particular stages of the EDP that relatively lower-achieving students struggled to understand in general, and stages of the EDP that students may have been inclined to skip or use out of order.

During our analysis of semi-structured qualitative interviews with students related to the EDP interviews, students expressed many of the same findings that we observed using distractor analysis-that is, that there were certain stages of the EDP that relatively lowerachieving students struggled to understand, and stages of the EDP that students may have been inclined to skip or use out of order. For example, in our distractor analysis of item 14, which asked students to identify relevant details to communicate to a company manager, we observed that students had some difficulty determining the specific details that would be most relevant for the specified customer. Results from student interviews reflected a similar finding. Specifically, students' responses to the interview questions suggested that students have a general grasp of what is needed to communicate with a client, but that this step is difficult. Further, in our distractor analysis of item 19, which asked students to identify an activity that reflects the Evaluate stage of the EDP, we found that students had some difficulty in distinguishing the unique characteristics of this stage from other stages in the EDP. Student responses during the interviews often indicated an understanding of the purpose of the Evaluate stage, which is to choose a design based on how likely it is to meet the requirements. However, some students demonstrated confusion with regard to the activities that correspond to this stage.

Our distractor analysis results also revealed stages of the EDP that students were inclined to skip over when considering a design challenge. In particular, our analyses of item 7 and item 12 revealed that students who did not select the correct answer choice experienced confusion related to the appropriate sequence of EDP stages. Students expressed similar difficulties during our interviews. For example, several students expressed confusion between the Prototype and Test stage and other stages in the EDP. Furthermore, student responses suggested that their confusion between EDP stages is often due to their desire to quickly move through the stages in order to create a prototypesimilar to our distractor analysis results for item 7 and item 12.

The results from our comparison of patterns of student answer choices across teachers were also interesting. Although all of the teachers who participated in our study received the same professional development with regard to the experimental curriculum and all of the students participated in the same curriculum, we observed different patterns of answer choices on the items for which there were significant differences in student achievement. In other words, students who selected incorrect responses appeared to do so for different reasons across classrooms. These results provide a starting point for additional research on differences in student achievement related to the EDP, and how achievement differences correspond to various misconceptions.

\section{Implications for engineering education}

Several scholars have explored the role of the EDP as a key component of student proficiency in engineering (e.g., Kelly 2014; Wendell and Kolodner 2014), particularly within the context of problem-based and/or project-based instruction (Marra, Jonassen, Palmer, \& Luft, 2014; e.g., Kolmos and De Graff 2014). Accordingly, our results suggest that distractor analysis is a valuable tool that can provide researchers and practitioners with insight into the development of students' understanding of the EDP that can inform curriculum development and instructional 
practices. As we noted earlier, although several researchers have applied distractor analysis techniques to science education (Herrmann-Abell and DeBoer 2011, 2014; Wind and Gale 2015), researchers have not yet used distractor analysis to explore students' responses to $\mathrm{MC}$ items in the context of engineering education. Accordingly, our study provides initial insight into the use of distractor analysis as a method for exploring students' conceptualizations of engineering concepts. When contrasted with other forms of engineering assessment that are intended to help researchers and practitioners understand students' conceptualizations of the EDP, such as engineering notebooks, distractor analysis of $\mathrm{MC}$ items is a promising alternative. In particular, when used with purposefully constructed MC items, distractor analysis techniques can provide researchers and practitioners with a potentially less time-consuming method by which to gain insight into students' understanding of engineering concepts. This approach is particularly promising when other approaches to assessing student understanding of the EDP are not practical. The approach that we demonstrated in our study can provide insight into student conceptualizations of EDP stages beyond the typical "correct/ incorrect" scoring procedures for MC assessments.

Our findings are particularly meaningful in light of recent emphases on engineering design as a key component of student proficiency in the integrated Science, Technology, Engineering, and Mathematics (STEM) disciplines (e.g., Borgford-Parnell et al. 2010; Cardella et al. 2008; Kelly 2014; Kolmos and De Graff 2014). Furthermore, current STEM curricula in the USA emphasize student proficiency in engineering as a key component of college and career readiness (Auyang 2004; Carr et al. 2012). Despite the importance placed on engineering education in policy and practice, there has been relatively limited attention in research on the development of psychometrically sound engineering assessment techniques. In this study, we illustrated a method that researchers and practitioners can use to explore the results from $\mathrm{MC}$ engineering assessments from a diagnostic perspective. In practice, researchers and practitioners can apply the techniques that we illustrated here to other MC assessments of engineering concepts. As we observed in this study, such analyses can provide insight into student conceptualizations of engineering concepts.

Furthermore, researchers and practitioners can use the distractor analysis techniques that we illustrated in this study as a diagnostic tool to evaluate the performance of distractors in MC items in order to improve assessment practices in engineering education. For example, distractor analysis techniques can help researchers and practitioners identify individual distractors in $\mathrm{MC}$ items that are not providing useful diagnostic information, or that might be eliciting confusion from otherwise high-achieving students. For example, evidence that none or very few of a sample of students selected a particular distractor may suggest that the answer choice is not helpful for distinguishing among students with different levels of understanding for a particular concept. Furthermore, evidence that otherwise high-achieving students are most attracted to a distractor rather than the correct answer choice may suggest a need to revise the distractor so that it does not introduce unnecessary confusion. Likewise, as we observed in our analysis of item 9, evidence that the lowestachieving students are most attracted to the correct answer, while students with higher levels of achievement select distractors may suggest confusion related to particular distractors that may encourage guessing from low-achieving students.

Finally, our results suggest that researchers and practitioners can use distractor analysis techniques as a diagnostic tool for exploring differences among subgroups of students in terms of their conceptualization of the EDP. In this study, we used distractor analysis techniques to investigate differences in patterns of students' responses within different classrooms for items on which we observed differences in student performance. This approach allowed us to understand the degree to which differences in students' conceptualizations of the EDP within these subgroups may have contributed to differences in their performance on the MC items. In practice, researchers and practitioners can use distractor analysis techniques to explore how achievement differences between other subgroups of students, such as demographic subgroups, may be related to different patterns of alternate conceptions that are reflected in $\mathrm{MC}$ item distractors.

\section{Implications for research on distractor analysis}

Our study also has implications for research on distractor analysis in general. Although several researchers have used distractor analysis techniques to explore differences over time (Wind and Gale 2015) and between grade levels (Herrmann-Abell and DeBoer 2011), researchers have not fully considered the use of distractor analysis as a supplementary tool to explore differences in student performance at the item level related to student subgroups. In this study, we illustrated a procedure for combining evidence of differential performance between student subgroups on individual items with distractor analysis in order to uncover potential contributing factors to achievement differences. This approach provides insight into differences in student performance across subgroups in terms of specific answer choices that reflect different aspects of students' understanding and application of a particular concept, such as stages of the EDP. Researchers can use the results of such analyses to inform instructional decisions in order to focus on specific concepts without the use 
of more time-intensive performance assessment procedures.

\section{Limitations and directions for future research}

Our study has several limitations that researchers and practitioners should consider before generalizing the results to other contexts. First, we conducted our analyses using data from seventh grade students who participated in an experimental middle grades engineering curriculum project in the USA. Likewise, we used data from one MC engineering assessment instrument. Our student sample and assessment instrument may not reflect the characteristics of other student populations and MC engineering assessments. Accordingly, we encourage researchers and practitioners to consider the alignment between the student sample and instrument that we used in this study and other populations of students and assessment instruments before generalizing our results. In future studies, researchers could explore the use of distractor analysis in other engineering education contexts in order to provide additional insight into the generalizability of our findings. In particular, we encourage researchers to explore the generalizability of our findings to larger samples of students than the sample that we included in this analysis. Along the same lines, we encourage researchers to conduct additional mixed-methods studies in which they explore student the alignment between student conceptualizations of the EDP and their specific answer choices to $\mathrm{MC}$ items. Specifically, researchers might use a sequential exploratory mixed-methods design in which they begin with a distractor analysis, and design qualitative data collection to purposefully target students who selected particular answer choices. Such analyses would provide additional insight into the interpretation of distractor analysis as a method for exploring student understanding of the EDP.

Second, it is important to note that we focused on one type of distractor analysis. Other types of distractor analysis, including methods based on cognitive diagnostic models or other distractor analysis models, may provide different information about students' response patterns. In future studies, researchers should apply distractor analysis techniques besides the methods that we used in this study to MC engineering assessments.

It is also important to note that the degree to which one can gain insight into students' conceptualizations of the EDP using distractor analysis is somewhat limited compared to other approaches, such as qualitative analyses of engineering design notebooks and interviews with students. Nonetheless, our findings suggest that distractor analysis provides valuable initial insight into student conceptualizations of the EDP that can serve as a starting place from which to inform additional investigations. In future studies, we encourage researchers to consider other forms of mixed-methods designs in which they combine distractor analysis techniques with qualitative methods in order to more fully understand students' conceptualizations of the EDP.

Finally, it is important to note that we used distractor analysis techniques to explore students' understanding of the EDP. We did not directly explore how teachers could use the results from such analyses to inform their approach and pedagogy, or the effectiveness of distractor analysis results for this purpose. In future studies, researchers could explore the implications of using distractor analysis results to inform teaching practices.

\section{Endnotes}

${ }^{1}$ This study was approved by the IRB at Georgia Institute of Technology, protocol number H13201.

\section{Additional file}

Additional file 1: Background information about the Engineering Design Process assessment. (DOCX 23 kb)

\section{Acknowledgements \\ The authors wish to acknowledge Dr. Jessica D. Gale and Dr. Sunni Newton for their assistance with data collection. \\ Funding \\ This research was supported by a grant from the National Science Foundation, grant \# 1238089. Any opinions, findings, and conclusions or recommendations expressed in these materials are those of the author(s) and do not necessarily reflect the views of the National Science Foundation.}

Availability of data and materials

Please contact the corresponding author for data requests.

\section{Authors' contributions}

SW, MA, JL, and RM designed the assessments used to collect data for this study. SW proposed the research questions and analytic approach, analyzed the data, and drafted most of the manuscript sections. AA assisted with the literature review and construction of the distractor analysis plots. MA, JL, and RM offered comments, revised the manuscript, and provided editorial suggestions. All of the authors read and approved of the final manuscript.

\section{Ethics approval and consent to participate}

The Institutional Review Board for Georgia Institute of Technology has approved the study reported in this manuscript. Parental consent and student assent procedure are in place per IRB requirement. The IRB protocol identification number is \#H13201. Additional documentation related to IRB approval is available from the corresponding author.

\section{Consent for publication}

Not applicable.

\section{Competing interests}

The authors declare that they have no competing interests.

\section{Publisher's Note}

Springer Nature remains neutral with regard to jurisdictional claims in published maps and institutional affiliations. 


\section{Author details}

'Educational Studies in Psychology, Research Methodology, and Counseling, The University of Alabama, Box 870231, Tuscaloosa 35487, AL, USA. ${ }^{2}$ Georgia Institute of Technology, Atlanta, GA, USA.

\section{Received: 19 June 2018 Accepted: 20 December 2018}

Published online: 23 January 2019

\section{References}

Adams, R. S., Turns, J., \& Atman, C. J. (2003). Education effective engineering designers: the role of reflective practice. Design Studies, 24(3), 275-294.

Alemdar, M., Lingle, J. A., Moore, R., \& Wind, S. A. (2017a). Developing an engineering design process assessment using think-aloud interviews. International Journal of Engineering Education, 33(1(B)), 441-452.

Alemdar, M., Lingle, J. A., Newton, S., Moore, R., Rosen, J., \& Usselman, M. (2017b). Students' perceptions of middle school STEM innovation and design course. In Proceedings of the ASEE 2017 Annual Conference. Columbus, OH.

Atman, C. J., Eris, O., McDonnell, J., Cardella, M. E., \& Borgford-Parnell, J. L. (2014). Engineering design education: research, practice, and examples that link the two. In J. Aditya \& B. M. Olds (Eds.), Cambridge handbook of engineering education research (pp. 201-225). Cambridge: Cambridge University Press.

Auyang, S. Y. (2004). Engineering — an endless frontier. Cambridge, MA: Harvard University Press.

Borgford-Parnell, J., Deibel, K., \& Atman, C. J. (2010). From engineering design research to engineering pedagogy: bringing research results directly to the students. International Journal of Engineering Education, 26(4), 748-759.

Briggs, D. C., Alonzo, A. C., Schwab, C., \& Wilson, M. (2006). Diagnostic assessment with ordered multiple-choice items. Educational Assessment, 11(1), 33-63 https://doi.org/10.1207/s15326977ea1101_2.

Carberry, A. R., Lee, H.-S., \& Ohland, M. W. (2010). Measuring engineering design self-efficacy. Journal of Engineering Education, 99(1), 71-79 https://doi.org/10. 1002/j.2168-9830.2010.tb01043.x.

Cardella, M., Atman, C. J., Turns, J., \& Adams, R. (2008). Students with differing design processes as freshmen: case studies on change. International Journal of Engineering Education, 24(2), 246-259.

Carr, R. L., Bennett, L. D., \& Strobel, J. (2012). Engineering in the K-12 STEM standards of the 50 U.S. states: an analysis of presence and extent. Journal of Engineering Education, 101(3), 1-26.

Creswell, J. W., \& Plano Clark, V. L. (2011). Designing and conducting mixed methods research. Thousand Oaks, CA: Sage.

de la Torre, J. (2009). A cognitive diagnosis model for cognitively based multiplechoice options. Applied Psychological Measurement, 33(3), 163-183 https://doi. org/10.1177/0146621608320523.

Eliot, M., \& Turns, J. (2011). Constructing professional portfolios: sense-making and professional identity development for engineer undergraduates. Journal of Engineering Education, 100(4), 630-654.

Gierl, M. J., Bulut, O., Guo, Q., \& Zhang, X. (2017). Developing, analyzing, and using distractors for multiple-choice tests in education: a comprehensive review. Review of Educational Research, 87(6), 1082-1116.

Haladyna, G., \& Rodriguez, M. C. (2013). Developing and validating test items. New York, NY: Routledge.

Herrmann-Abell, C. F., \& DeBoer, G. E. (2011). Using distractor-driven standards-based multiple-choice assessments and Rasch modeling to investigate hierarchies of chemistry misconceptions and detect structural problems with individual items. Chemistry Education Research and Practice, 12(2), 184-192.

Herrmann-Abell, C. F., \& DeBoer, G. E. (2014). Developing and using distractordriven multiple-choice assessments aligned to ideas about energy forms, transformation, transfer, and conservation. In R. F. Chen, A. Eisenkraft, D. Fortus, J. Krajcik, K. Neumann, J. Nordine, \& A. Scheff (Eds.), Teaching and learning of energy in K-12 education (pp. 103-133). Springer.

Hestenes, D., Wells, M., \& Swackhamer, G. (1992). Force concept inventory. The Physics Teacher, 30(3), 141-158.

Jorion, N., Gane, B. D., James, K., Schroeder, L., DiBello, L. V., \& Pellegrino, J. W. (2015). An analytic framework for evaluating the validity of concept inventory claims. Journal of Engineering Education, 104(4), 454-496.

Junker, B. W., \& Sijtsma, K. (2001). Cognitive assessment models with few assumptions, and connections with nonparametric item response theory. Applied Psychological Measurement, 25(3), 258-272 https://doi.org/10.1177/ 01466210122032064.
Kelly, A. F. (2014). Design-based research in engineering education: current state and next steps. In A. Johri \& B. M. Olds (Eds.), Handbook of engineering education research (pp. 497-518). New York, NY: Cambridge University Press.

Klassen, S. (2006). Contextual assessment in science education: background, issues, and policy. Science Education, 90(5), 820-851.

Kolmos, A., \& De Graff, E. (2014). Problem-based and project-based learning in engineering education. In Handbook of engineering education research (pp. 797-518). New York, NY: Cambridge University Press.

Kong, Y., Diefes-Dux, H. A., Rodgers, K. J., Douglas, K. A., \& Madhavan, K. (2014). Development and validation of a nano size and scale instrument (NSSI). In 2014 IEEE Frontiers in Education Conference (FIE) (pp. 1-4). IEEE https://doi.org/ 10.1109/FIE.2014.7044332.

Linacre, J. M. (1989). Many-facet Rasch measurement. Chicago, IL: MESA Press.

Linacre, J. M. (1994). Sample size and item calibration stability. Rasch Measurement Transactions, 7(4), 328.

Linacre, J. M. (1998). Structure in Rasch residuals: Why principal components analysis (PCA)? Rasch Measurement Transactions, 12(2), 636.

Linacre, J. M. (2015). Facets Rasch measurement (version 3.71.4). Chicago, IL: Winsteps.com

Marra, R., Jonassen, D. H., Palmer, B., \& Luft, S. (2014). Why problem-based learning works: Theoretical foundations. Journal on Excellence in College Teaching, 25(3\&4), 221-238.

Miles, M. B., Huberman, A. M., \& Saldana, J. (2014). Qualitative data analysis (3rd ed.). Thousand Oaks, CA: Sage.

Mourtes, N. J. (1999). Portfolio assessment in aerodynamics. Journal of Engineering Education, 88, 223-229.

NGSS Lead States. (2013). Next generation science standards: For states, by states. Washington, DC: The National Academies Press.

Purzer, S. (2011). The relationship between team discourse, self-efficacy, and individual achievement: a sequential mixed-methods study. Journal of Engineering Education, 100(4), 655-679.

Rasch, G. (1960). Probabilistic models for some intelligence and achievement tests (expanded edition, 1980). Chicago: University of Chicago Press.

Reckase, M. D. (1979). Unifactor latent trait models applied to multifactor tests: Results and implications. Journal of Educational and Behavioral Statistics, 4(3), 207-230 https://doi.org/10.3102/10769986004003207.

Sengupta, S., Ergas, S., Cunningham, J., Goel, R., Feldman, A., \& Chen, Y.-H. (2017). Concept inventory for fundamentals of environmental engineering courses: concept inventory development and testing. Environmental Engineering Science, 34(12), 895-907.

Smith, R. M. (2004). Fit analysis in latent trait models. In E. V. Smith \& R. M. Smith (Eds.), Introduction to Rasch measurement (pp. 73-92). Maple Grove, MN: JAM Press.

Sobek, D., II. (2002). Use of journals to evaluate student design processes. Montreal: Paper presented at the American Society for Engineering Education conference.

Thissen, D., Steinberg, L., \& Fitzpatrick, A. R. (1989). Multiple-choice models: the distractors are also part of the item. Journal of Educational Measurement, 26(2), 161-176

Treagust, D. F. (1995). Diagnostic assessment of students' science knowledge. In S. M. Glynn \& R. Duit (Eds.), Learning science in the schools: Research reforming practice (pp. 327-346). Mahwah, NJ: Erlbaum.

Wage, K. E., Buck, J. R., Wright, C. H. G., \& Welch, T. B. (2005). The signals and systems concept inventory. IEEE Transactions on Education, 48(3), 448-461.

Wang, W.-c. (1998). Rasch analysis of distractors in multiple-choice items. Journal of Outcome Measurement, 2(1), 43-65.

Williams, J. M. (2001). The engineering portfolio: communication, reflection, and student learning outcomes assessment. International Journal of Engineering Education, 18(2), 199-207.

Wilson, M. (1992). The ordered partition model: an extension of the partial credit model. Applied Psychological Measurement, 16(4), 309-325 https://doi.org/10. 1177/014662169201600401.

Wind, S. A., Alemdar, M., Gale, J. D., Lingle, J. A., \& Moore, R. (2017). Developing an engineering design process assessment using mixed methods: an illustration with Rasch measurement theory and cognitive interviews. Journal of Applied Measurement, 18(2), 100-121.

Wind, S. A., \& Gale, J. D. (2015). Diagnostic opportunities using Rasch measurement in the context of a misconceptions-based physical science assessment. Science Education, 99(4), 721-741 https://doi.org/10.1002/sce. 21172

Wright, B. D., \& Stone, M. H. (1979). Best Test Design. Chicago, IL: MESA Press.

Wu, M., \& Adams, R. J. (2013). Properties of Rasch residual fit statistics. Journal of Applied Measurement, 14(4), 339-355. 